\title{
Probing the nature of AX J0043-737: Not an 87 ms pulsar in the Small Magellanic Cloud
}

\author{
C. Maitra ${ }^{1,2}$, J. Ballet ${ }^{2}$, P. Esposito ${ }^{3}$, F. Haberl ${ }^{1}$, A. Tiengo ${ }^{4,5,6}$, M. D. Filipović ${ }^{7}$, and F. Acero ${ }^{2}$ \\ ${ }^{1}$ Max-Planck-Institut für extraterrestrische Physik, Giessenbachstraße, 85748 Garching, Germany \\ e-mail: cmaitra@mpe.mpg.de \\ ${ }^{2}$ Laboratoire AIM, CEA/DRF - CNRS - Université Paris Diderot, IRFU/DAp, CEA-Saclay, 91191 Gif-sur-Yvette, France \\ 3 Anton Pannekoek Institute for Astronomy, University of Amsterdam, Postbus 94249, 1090 GE Amsterdam, The Netherlands \\ ${ }^{4}$ Scuola Universitaria Superiore IUSS Pavia, piazza della Vittoria 15, 27100 Pavia, Italy \\ ${ }^{5}$ INAF, Istituto Nazionale di Astrofisica, IASF-Milano, via E. Bassini 15, 20133 Milano, Italy \\ ${ }^{6}$ INFN, Istituto Nazionale di Fisica Nucleare, Sezione di Pavia, via A. Bassi 6, 27100 Pavia, Italy \\ 7 Western Sydney University, Locked Bag 1797, Penrith South DC, NSW 1797, Australia
}

Received 6 November 2017 / Accepted 4 January 2018

\begin{abstract}
Aims. AX J0043-737 is a source in the ASCA catalogue whose nature is uncertain. It is most commonly classified as a Crab-like pulsar in the Small Magellanic Cloud (SMC) following apparent detection of pulsations at $\sim 87 \mathrm{~ms}$ from a single ASCA observation. A follow-up ASCA observation was not able to confirm this, and the X-ray detection of the source has not been reported since.

Methods. We studied the nature of the source with a dedicated XMM-Newton observation. We ascertained the source position, searched for the most probable counterpart, and studied the X-ray spectrum. We also analysed other archival observations with the source in the field of view to study its long-term variability.

Results. With the good position localisation capability of XMM-Newton, we identify the counterpart of the source as MQS J004241.66-734041.3, an active galactic nucleus (AGN) behind the SMC at a redshift of 0.95. The X-ray spectrum can be fitted with an absorbed power law with a photon-index of $\Gamma=1.7$, which is consistent with that expected from AGNs. By comparing the current XMM-Newton observation with an archival XMM-Newton and two other ASCA observations of the source, we find signatures of long-term variability, another common phenomenon in AGNs. All of the above are consistent with AX J0043-737 being an AGN behind the SMC.
\end{abstract}

Key words. Magellanic Clouds - quasars: general - X-rays: galaxies - pulsars: individual: AX J0043-737

\section{Introduction}

The source AX J0043-737 is an elusive object in the ASCA catalogue that deserves attention; its nature remains uncertain, even 17 years after its first detection. The source, alternately known as SXP0.09, is most commonly classified as a Crab-like pulsar in the Small Magellanic Cloud (SMC), although an X-ray binary or an active galactic nucleus (AGN) behind the SMC have also been suggested as the possible nature of the source.

AX J0043-737 was discovered during ASCA observations of the SMC on 10-11 May 1999 (Yokogawa \& Koyama 2000; Yokogawa et al. 2003). Pulsations at $87.58073 \pm 0.00004 \mathrm{~ms}$ were reported from the source making it the fastest pulsar in the SMC. The significance of the detection was however marginal at $99.98 \%$. A follow-up ASCA observation with a longer exposure could not confirm the pulsations. A possible explanation was the lower count rate and larger background fraction in the latter observation. Confirmation of the pulsations with a higher significance, hence inferring the nature of the source, was thus highly solicited. The X-ray spectrum of the source was consistent with an absorbed power-law of the photon index $(\Gamma)$ of $\sim 1.7$. The inferred X-ray luminosity at the distance of the SMC $(60 \mathrm{kpc})$ was $8.6 \times 10^{34} \mathrm{erg} \mathrm{s}^{-1}$.

An X-ray detection of the source has not been reported since. AX J0043-737 is still quoted in the literature of pulsars in the SMC as a possible Crab-like pulsar, the fastest in the SMC (e.g.: Majid et al. 2004; Coe et al. 2005; Liu et al. 2005; Galache et al. 2008; Rajoelimanana et al. 2011; Christodoulou et al. 2016). In addition, no radio-continuum source was ever found in the ATCA surveys (Crawford et al. 2001; Wong et al. 2012). In this paper we report a dedicated XMM-Newton observation of AXJ0043-737. With the precise position localisation capability of XMM-Newton and its good sensitivity, we classify AX J0043-737 as an AGN behind the SMC, breaking the decade-long mystery surrounding the nature of the source. Using other archival observations we also study its long-term variability. Section 2 describes the observations and data reduction. Section 3 provides the results and a discussion. In Sect. 4 we outline and summarise our conclusions.

\section{Observations and data reduction}

AX J0043-737 was observed with XMM-Newton on 16 October, 2015 (OBSID:0764780201), for $40 \mathrm{ks}$. In addition we found an archival XMM observation where the source was in the field of view, and also analysed the two ASCA observations of the source. The details of the observations are given in Table 1 .

XMM-Newton/EPIC (Strüder et al. 2001; Turner et al. 2001) observations were processed with the latest XMM-Newton data analysis software SAS version 16.1.0 ${ }^{1}$. Examination for periods of high background-flaring activity was performed by

\footnotetext{
Science Analysis Software (SAS): http://xmm.esac.esa.int/ sas/
} 
Table 1. Observations of AX J0043-737.

\begin{tabular}{cccccc}
\hline \hline Observation & ObsID & Exposure (ks) & Date (dd/mm/yyyy) & Data mode & Off-axis \\
\hline ASCA1 & 67021000 & 20 & $10 / 05 / 1999$ & Pulse height (GIS) & $2.7^{\prime}$ \\
ASCA2 & 48003000 & 60 & $04 / 07 / 2000$ & Pulse height (GIS) & $4.3^{\prime}$ \\
XMM1 & 0301170301 & 15 & $04 / 06 / 2006$ & FULL FRAME (PN) & $5^{\prime}$ \\
XMM2 & 0764780201 & 40 & $16 / 10 / 2015$ & SMALL WINDOW (PN) & $0^{\prime}$ \\
\hline
\end{tabular}

extracting light curves in the energy range of 7.0-15.0 keV and removing time intervals with background rates $\geq 8$ and $2.5 \mathrm{cts} \mathrm{ks}^{-1}$ arcmin $^{-2}$ for EPIC-PN and EPIC-MOS, respectively (Sturm et al. 2013). Event extraction was performed using the SAS task evselect applying the standard filtering criteria (\#XMMEA_EP \&\& PATTERN $<=4$ for EPIC-pn and \#XMMEA_EM \&\& PATTERN $<=12$ for EPIC-MOS).

ASCA observations (Tanaka et al. 1994) were analysed following standard procedures ${ }^{2}$. Only data from the gas-scintillation imaging proportional counters (GIS2 and GIS3) were used for this work, as the source fell in a CCD gap for the X-ray CCD detectors (SIS). Spectra were extracted from the screened events using the FTOOLS task xselect after applying standard filtering criteria.

\section{Results and discussion}

\subsection{X-ray position}

A maximum-likelihood source detection analysis was performed on the XMM-Newton/EPIC images to determine the X-ray position of AX J0043-737. Fifteen images were created from the EPIC cameras in five energy bands as given: $1 \rightarrow(0.2-0.5) \mathrm{keV}$, $2 \rightarrow(0.5-1.0) \mathrm{keV}, 3 \rightarrow(1.0-2.0) \mathrm{keV}, 4 \rightarrow(2.0-4.5) \mathrm{keV}, 5 \rightarrow$ (4.5-12.0) keV (Watson et al. 2009; Sturm et al. 2013). Source detection was performed simultaneously on all the images using the SAS task edetect_chain. Astrometric boresight correction was performed with the task eposcorr accounting for a linear shift. The reference sources were a catalogue of background AGNs with known redshifts as well as sources selected using ALLWISE mid-infrared colour-selection criteria (Mateos et al. 2012; Secrest et al. 2015).

From the two XMM-Newton observations the error-weighted mean of the position was RA $=00^{\mathrm{h}} 42^{\mathrm{m}} 41^{\mathrm{s}} .67$ and Dec $=$ $-73^{\circ} 40^{\prime} 40^{\prime} 8$ (J2000) with a $1 \sigma$ statistical uncertainty of $0.27^{\prime \prime}$. The positional error is usually dominated by systematic astrometric uncertainties. Therefore, we added a systematic error of $0.37^{\prime \prime}$ in quadrature (Rosen et al. 2016a). The improved position of the source is $\sim 0.3$ arcmin away from the original value as found from the ASCA observations. With the new position we found that AX J0043-737 is coincident with XMMU J004241.5-734041 in the XMM-Newton point-source catalogue of the SMC (Sturm et al. 2013), and XMMU J004241.5-734039 in the 3XMM-DR6 catalogue (Rosen et al. 2016b).

\subsection{Source identification and search for counterpart}

XMMU J004241.5-734041 is classified as an AGN candidate in the SMC point-source catalogue from its optical and X-ray colours (Sturm et al. 2013). Recently, the all-sky catalogue of 1.4 million AGNs of Secrest et al. (2015), and the Half-Million Quasars catalogue (HMQ) and MILLIQUAS catalogue of Flesch (2015, 2017) have increased the number of AGNs behind the SMC by several orders of magnitude. To verify the credibility

2 https://heasarc.gsfc.nasa.gov/docs/asca/abc/abc.html/ of the classification of AX J0043-737 as an AGN candidate, we correlated the source with the above mentioned catalogues with an angular separation of

$r \leq 3.439 \times \sqrt{\sigma_{\mathrm{X}}^{2}+\sigma_{\text {catalogue }}^{2}}=3.439 \sigma$.

For a Rayleigh distribution this corresponds to a $3 \sigma$ completeness. We found the most probable counterpart (best match) as MQS J004241.66-734041.3 (catalogued in Flesch 2015, 2017) within $0.49^{\prime \prime}$ of the source position. To calculate the probability of chance coincidence we calculated the total number of AGNs identified in X-rays from the AGN catalogues within the XMMNewton survey area of the SMC. This resulted in a total of 270 sources being identified within $6.67 \mathrm{deg}^{2}$. Considering the area of the error circle corresponding to Eq. (1), and that the AGNs are distributed homogeneously within the survey area, the probability of chance coincidence is $8.4 \times 10^{-6}$.

MQS J004241.66-734041 was spectroscopically confirmed by Kozłowski et al. (2013). Kozłowski et al. (2013) selected candidates from the Optical Gravitational Lensing Experiment (OGLE-III) based on their optical variability and/or X-ray properties (OGLE-III ID of the source: smc128.4.5190). The $V, R$, and $I$ magnitudes of the source are 20.18, 19.99, and 19.91, respectively. The redshift is $z=0.95$ indicating a luminosity distance of $6.2 \mathrm{Gpc}$ to the AGN assuming standard cosmological parameters.

In addition, we also looked for a possible counterpart in the Magellanic Clouds Photometric Survey (MCPS; Zaritsky et al. 2002). The closest optical counterpart has $V$ and $I$ magnitudes consistent with MQS J004241.66-734041.3. There is no other bright and statistically acceptable optical counterpart. This is further ascertained in Fig. 1, which shows the finding chart for AX J0043-737. This was constructed using the $J, H$, and $K$ band VISTA images which are publicly available ${ }^{3}$. Considering these we conclude that AX J0043-737 is the X-ray counterpart of MQS J004241.66-734041.3, an AGN behind the SMC.

\subsection{Timing analysis}

The PN data of XMM2 were taken in small-window mode, providing a time resolution of $5.7 \mathrm{~ms}$. We searched the PN data in the $0.3-10 \mathrm{keV}$ energy range for possible periodic signals using a Fourier transform in the frequency range spanning $\sim 0.00002-$ $88.2 \mathrm{~Hz}$. No significant signal was found, with a $3 \sigma$ upper limit on the pulsed fraction of $\sim 40 \%$ (assuming a sinusoidal profile). We also performed a search with the $Z^{2}$ test in a small interval bracketing the ASCA tentative detection $(85-90 \mathrm{~ms})$. The most prominent peak occurs at $87.53022(2) \mathrm{ms}$, with a $8 \times 10^{-7}$ singletrial probability. When the $\sim 6 \times 10^{4}$ independent period searches are taken into account, its statistical significance is rather low $(\lesssim 2 \sigma)$. Since the significance of this signal entirely relies on the marginal ASCA1 detection, which was not confirmed by the ASCA2 data set, we conclude that there is no evidence in the

3 http://horus.roe.ac.uk/vsa/index.html 


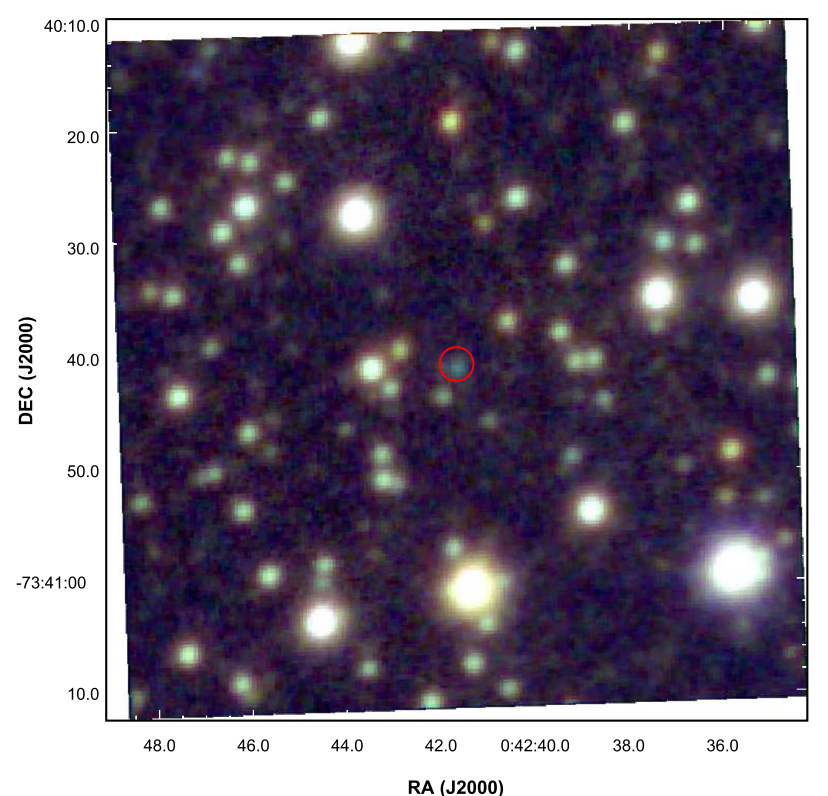

Fig. 1. VISTA image of the region around AX J0043-737. The image covers $1^{\prime} \times 1^{\prime}$ sky area. The RGB colours correspond to the $J, H$, and $K$ VISTA filters, respectively. The red circle is centred on the $X M M$ Newton position of AX J0043-737 with a radius corresponding to a $3.439 \sigma$ confidence error circle (including statistical and systematic errors.)

PN data for any periodic signal, not even restricting the search around the ASCA value.

\subsection{Spectral analysis}

For the XMM-Newton observations circular regions with radii of $20^{\prime \prime}$ and annular regions with inner and outer radii of $40^{\prime \prime}$ and $60^{\prime \prime}$, respectively, were used for the source and background extraction. The SAS tasks rmfgen and arfgen were used to create the redistribution matrices and ancillary files for the spectral analysis. The spectra were binned to achieve 25 counts in each bin and to not oversample the intrinsic energy resolution by a factor larger than 3 .

For the spectral extraction of the two ASCA observations, a different prescription was required owing to the poor angular resolution of the ASCA X-ray telescopes (XRT). The PSF of XRT has a relatively sharp core (FWHM of $\sim 50^{\prime \prime}$ ) but broad wings (with half-power diameter of $3^{\prime}$ ). The PSF is dependent on the energy, the angle from the optical axis, and the azimuthal angle of the source position. The GIS in addition has its own PSF, which is comparable in width to that of the XRT albeit with a different shape leading to an additional spatial broadening 4 .

Following this, a circular region of radius $4^{\prime}$ was used to extract the source spectrum. A large annular region with inner and outer radii of $8^{\prime}$ and $10^{\prime}$ surrounding the source was used to extract the background spectrum. However the images extracted from the combined GIS (GIS2 and GIS3) showed the presence of another source within the extraction region of AX J0043-737. The contaminating source was identified as XMMU J004207.7-734503, a candidate HMXB in the SMC point-source catalogue (Sturm et al. 2013). This prompted us to fit the spectra of the two sources simultaneously in the ASCA observations, after taking into account the contamination from

\footnotetext{
4 https://heasarc.gsfc.nasa.gov/docs/asca/abc/
} node11.html
Table 2. Spectral parameters of AX J0043-737 and XMMU J004207.7-734503 from XMM2.

\begin{tabular}{cc}
\hline \hline Parameters & Value \\
\hline AX J0043-737 & \\
\hline SMC $N_{\mathrm{H}}\left(10^{22}\right.$ atoms cm $\left.{ }^{-2}\right)$ & $0.16 \pm 0.07$ \\
$\Gamma$ & $1.72 \pm 0.19$ \\
Flux $^{a}(0.5-8.0 \mathrm{keV})$ & $6.6 \pm 0.7$ \\
Flux (unabsorbed $)^{a}(0.5-8.0 \mathrm{keV})$ & $8.1 \pm 1.0$ \\
\hline XMMU J004207.7-734503 \\
\left.\hline${\mathrm{SMC} N_{\mathrm{H}}\left(10^{22} \text { atoms cm }\right.}^{-2}\right)$ & $0.75 \pm 0.34$ \\
$\Gamma$ & $1.06 \pm 0.16$ \\
Flux $^{a}(0.5-8.0 \mathrm{keV})$ & $25.7 \pm 2.4$ \\
Flux (unabsorbed $)^{a}(0.5-8.0 \mathrm{keV})$ & $28.3 \pm 2.5$ \\
\hline
\end{tabular}

Notes. Errors are quoted at $90 \%$ confidence. (a) Flux in units of $10^{-14} \mathrm{erg} \mathrm{cm}^{-2} \mathrm{~s}^{-1}$.

one source into the other. In order to do this, we extracted the spectra from both sources using smaller radii to minimise the contamination $\left(2^{\prime}\right)$. Furthermore we accounted for the percentage spillover from each source to the other within the source extraction regions in the following manner: We modeled the ASCA PSF at the source positions from ASCA observations of Cygnus X-1 taken at different positions with respect to the optical axis. These are available in the energy range of 1$10 \mathrm{keV}$ at intervals of $1 \mathrm{keV}^{5}$. In order to take into account the energy dependence, we reconstructed the PSF at the position of each source by adding the PSFs in different energy bands weighed by the spectrum of the respective sources. For AX J0043-737, a power-law with $\Gamma=1.7$ was used. For the HMXB candidate XMMU J004207.7-734503, $\Gamma=1.06$ was used. This was obtained by fitting the spectrum extracted from XMM1 with an absorbed power-law model (Table 2). Comparing the PSFs resulted in a total spillover fraction of $11.06 \%$ from XMMU J004207.7-734503 into AX J0043-737 and 9.76\% from AX J0043-737 into XMMU J004207.7-734503 in the energy range of $0.8-7 \mathrm{keV}$ within the respective source-extraction regions. The redistribution matrices for the GIS data were obtained from the HEASARC calibration database ${ }^{6,7}$. The ancillary files were generated using the command, arf. The spectra were binned to achieve 25 counts in each bin.

X-ray spectral analysis was performed using the XSPEC fitting package, Version 12.8.1 (Arnaud 1996). In this section, we report the spectral parameters obtained by fitting XMM2. The total count rates in the source and background regions for XMM2 were $0.02,0.005,0.004,0.005,7.0 \times 10^{-4}$ and $6.0 \times 10^{-4} \mathrm{c} \mathrm{s}^{-1}$ for the PN, MOS1, and MOS2, respectively. To account for the photoelectric absorption by the interstellar gas, two components were used. The first one was fixed to the Galactic value of $6 \times 10^{20} \mathrm{~cm}^{-2}$ (Dickey \& Lockman 1990). The other one was left free to account for the absorption within the SMC. For the latter component, the metal abundances were fixed at 0.2 solar, as is typical in the SMC (Russell \& Dopita 1992). The atomic cross sections were adapted from Verner et al. (1996).

An absorbed power-law spectral model provided an acceptable fit with reduced $\chi^{2}$ of 1.1 for 31 d.o.f. (Fig. 2). The spectral

\footnotetext{
https://heasarc.gsfc.nasa.gov/docs/asca/abc/ node 11. html

6 ftp://legacy.gsfc.nasa.gov/caldb/data/asca/gis/cpf/ 95mar06/gis2v4_0.rmf

7 ftp://legacy.gsfc.nasa.gov/caldb/data/asca/gis/cpf/ 95mar06/gis3v4_0.rmf
} 


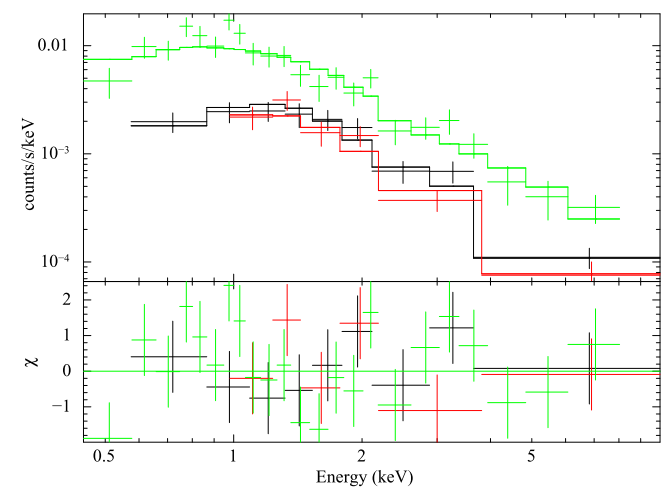

Fig. 2. Upper panel: simultaneous spectral fit of AX J0043-737 during XMM2 using spectra from all the XMM-Newton/EPIC cameras (PN in green, MOS1 in red and MOS2 in black) along with the best-fit model. Lower panel: residuals after the fit.

parameters are tabulated in Table 2. The X-ray spectra of AGNs can typically be described by a power law with photon index of $\Gamma \sim 1.75$ (e.g. Tozzi et al. 2006). The obtained $\Gamma$ of $\sim 1.7$ is compatible with AX J0043-737 being an AGN behind the $\mathrm{SMC}$. From the estimated luminosity distance, the determined absorption-corrected X-ray luminosity $(0.5-8.0 \mathrm{keV})$ is $1.9 \times$ $10^{44} \mathrm{erg} \mathrm{s}^{-1}$.

\subsection{Long-term variability}

The X-ray flux of AGNs varies on timescales ranging from hours to up to several years (see e.g. Ponti et al. 2012; Vagnetti et al. 2011). The three archival observations of the source (1 XMMNewton and 2 ASCA) provide the opportunity to probe the longterm variability (if any) of the source spanning 17 years. To test this we performed spectral analysis as described below.

We fitted AX J0043-737 and XMMU J004207.7-734503 together because they are blended in the ASCA data. We used a two-component power-law model (one corresponding to each source) and all the observations were fitted simultaneously. Each source was described by the same spectral parameters in all observations. Possible variations were parameterised by scale factors with respect to XMM2 (relative flux with respect to the best observation, XMM2). The other observations are not deep enough to test variability in the spectral shape and even if it existed, it would nearly always be associated with flux variability. For the ASCA observations the fraction of spillover from one source into the other was taken into account. The difference in $\chi^{2}$ with the scale factors fixed and left free is 40 for 3 d.o.f. This corresponds to a chance probability $<0.00001$ and is highly significant. Figure 3 shows a comparison of the variation of the scale factor over the observations for the two sources. AX J0043-737 shows a noticeable difference in the values especially between the ASCA and XMM-Newton observations. The corresponding values for XMMU J004207.7-734503 however do not show such a trend indicating that the variation is not due to differences in calibration between the two instruments. AX J0043-737 therefore exhibits signs of long-term variability typical for AGNs.

\section{Summary and conclusions}

With a dedicated XMM-Newton observation of AX J0043-737 we classify this object as an AGN behind the SMC. The source coincides with MQS J004241.66-734041.3 which lies at a redshift of 0.95 . The fit of a power-law model to the X-ray spectrum gives a spectral index typical for AGNs and has an

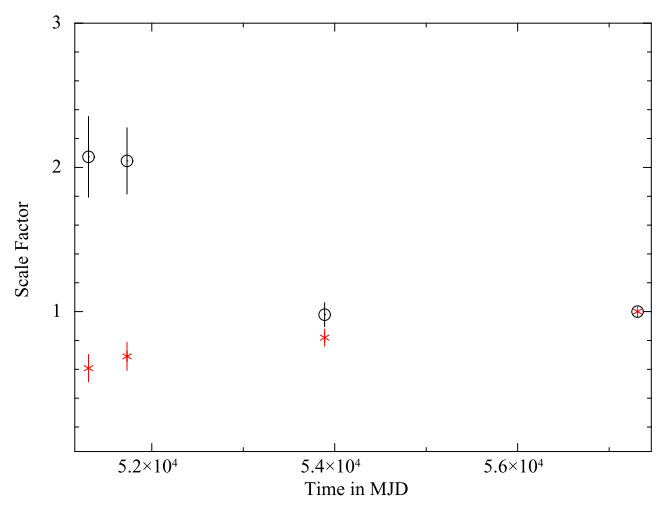

Fig. 3. Comparison of the scale factors (relative flux with respect to XMM2) for AX J0043-737 (in black circle) and XMMU J004207.7734503 (in red cross) with time in MJD.

absorption-corrected X-ray luminosity of $1.9 \times 10^{44} \mathrm{erg} \mathrm{s}^{-1}$ in the energy range of $0.5-8 \mathrm{keV}$. By comparing the archival observations spanning 17 years we find signatures of long-term variability. All of the above observations are consistent with the source being an AGN behind the SMC. More importantly, this answers the decade-long question on the nature of the source and rectifies its misidentification as an $87 \mathrm{~ms}$ pulsar in the SMC.

Acknowledgements. The XMM-Newton project is supported by the Bundesministerium für Wirtschaft und Technologie/Deutsches Zentrum für Luft-und Raumfahrt (BMWi/DLR, FKZ 50 OX 0001) and the Max-Planck Society. PE acknowledges funding in the framework of the NWO Vidi award A.2320.0076. We thank the referee for useful suggestions that significantly helped to improve the paper.

\section{References}

Arnaud, K. A. 1996, in Astronomical Data Analysis Software and Systems V, eds. G. H. Jacoby, \& J. Barnes, ASP Conf. Ser., 101, 17

Christodoulou, D. M., Laycock, S. G. T., Yang, J., \& Fingerman, S. 2016, ApJ, 829,30

Coe, M. J., Edge, W. R. T., Galache, J. L., \& McBride, V. A. 2005, MNRAS, 356,502

Crawford, F., Kaspi, V. M., Manchester, R. N., et al. 2001, ApJ, 553, 367

Dickey, J. M., \& Lockman, F. J. 1990, ARA\&A, 28, 215

Flesch, E. W. 2015, PASA, 32, e010

Flesch, E. W. 2017, VizieR Online Data Catalog, VII/277

Galache, J. L., Corbet, R. H. D., Coe, M. J., et al. 2008, ApJS, 177, 189

Kozłowski, S., Onken, C. A., Kochanek, C. S., et al. 2013, ApJ, 775, 92

Liu, Q. Z., van Paradijs, J., \& van den Heuvel, E. P. J. 2005, A\&A, 442, 1135

Majid, W. A., Lamb, R. C., \& Macomb, D. J. 2004, ApJ, 609, 133

Mateos, S., Alonso-Herrero, A., Carrera, F. J., et al. 2012, MNRAS, 426, 3271

Ponti, G., Papadakis, I., Bianchi, S., et al. 2012, A\&A, 542, A83

Rajoelimanana, A. F., Charles, P. A., \& Udalski, A. 2011, MNRAS, 413, 1600

Rosen, S. R., Webb, N. A., Watson, M. G., et al. 2016a, A\&A, 590, A1

Rosen, S. R., Webb, N. A., Watson, M. G., et al. 2016b, VizieR Online Data Catalog, IX/50

Russell, S. C., \& Dopita, M. A. 1992, ApJ, 384, 508

Secrest, N. J., Dudik, R. P., Dorland, B. N., et al. 2015, ApJS, 221, 12

Strüder, L., Briel, U., Dennerl, K., et al. 2001, A\&A, 365, L18

Sturm, R., Haberl, F., Pietsch, W., et al. 2013, A\&A, 558, A3

Tanaka, Y., Inoue, H., \& Holt, S. S. 1994, PASJ, 46, L37

Tozzi, P., Gilli, R., Mainieri, V., et al. 2006, A\&A, 451, 457

Turner, M. J. L., Abbey, A., Arnaud, M., et al. 2001, A\&A, 365, L27

Vagnetti, F., Turriziani, S., \& Trevese, D. 2011, A\&A, 536, A84

Verner, D. A., Ferland, G. J., Korista, K. T., \& Yakovlev, D. G. 1996, ApJ, 465, 487

Watson, M. G., Schröder, A. C., Fyfe, D., et al. 2009, A\&A, 493, 339

Wong, G. F., Crawford, E. J., Filipovic, M. D., et al. 2012, Serb. Astron. J., 184, 93

Yokogawa, J., Imanishi, K., Tsujimoto, M., Koyama, K., \& Nishiuchi, M. 2003, PASJ, 55, 161

Yokogawa, J., \& Koyama, K. 2000, IAU Circ., 7361

Zaritsky, D., Harris, J., Thompson, I. B., Grebel, E. K., \& Massey, P. 2002, AJ, 123,855 